\title{
AGROMETEOROLOGIA
}

Nota

\section{PERDA DE PRODUTIVIDADE POTENCIAL DA CULTURA DO SORGO NO ESTADO DE SÃO PAULO ${ }^{(1)}$}

\author{
FÁBIO RICARDO MARIN ${ }^{(2)}$; HÉLITON PANDORFI ${ }^{(3)}$; PAULO CÉSAR SENTELHAS ${ }^{(3,6)}$; \\ MARCELO BENTO PAES DE CAMARGO ${ }^{(4,6)}$; FERNANDO BRAZ TANGERINO HERNANDEZ ${ }^{(5)}$
}

\section{RESUMO}

O objetivo deste trabalho foi estimar a perda de produtividade potencial do sorgo em 36 épocas de semeadura, para quatro localidades do Estado de São Paulo: Manduri, Piracicaba, Ribeirão Preto e Ilha Solteira. As estimativas basearam-se nas chances de atendimento das exigências hídricas da cultura, utilizando-se do método de Doorenbos e Kassam/FAO, para estimativa da produtividade potencial e real. Foi necessário acrescentar um fator de correção com base na temperatura do ar para a obtenção de estimativas coerentes com o modelo. Utilizaram-se séries de dados climatológicos entre 10 e 40 anos. Foi possível identificar épocas de semeadura com menores riscos para o cultivo do sorgo e as chances de perda para cada época do ano. Em Manduri, Piracicaba, Ilha Solteira e Ribeirão Preto, os riscos de quebra de produção foram menores nas semeaduras entre 15-10 e 15-11, com quebras inferiores a 5\%. Em todas as localidades, foram observadas perdas inferiores a $5 \%$ para semeaduras realizadas em fevereiro.

Palavras-chave: Sorghum bicolor, balanço hídrico, época de semeadura, risco climático.

( $\left.{ }^{1}\right)$ Recebido para publicação em 5 de maio de 2005 e aceito em 9 de fevereiro de 2006.

$\left({ }^{2}\right)$ EMBRAPA Informática Agropecuária, Av. André Tosello, 209, 13083-886 Campinas (SP). E-mail: marin@cnptia.embrapa.br

$\left(^{3}\right)$ Departamento de Ciências Exatas, ESALQ/USP, Caixa Postal 9, 13418-900 Piracicaba (SP). E-mail: hpandorf@esalq.usp.br; pcsentel@esalq.usp.br

$\left({ }^{4}\right)$ Centro de Ecofisiologia e Biofísica, Instituto Agronômico, Av. Barão de Itapura, 1.481, Caixa Postal 28, 13020-902 Campinas (SP). E-mail: mcamargo@iac.sp.gov.br

$\left(^{5}\right)$ Departamento de Fitossanidade, Engenharia Rural e Solos, UNESP, Caixa Postal 31, 15385-000 Ilha Solteira (SP). E-mail: fbthtang@agr.feis.unesp.br

$\left({ }^{6}\right)$ Bolsista do CNPq. 


\section{ABSTRACT \\ POTENTIAL YIELD LOSS OF SORGHUM CROP IN THE STATE OF SÃO PAULO, BRAZIL}

This study was carried out to estimate the sorghum yield loss for 36 different sowing dates for four regions of the State of São Paulo, Brazil: Manduri, Piracicaba, Ribeirão Preto and Ilha Solteira. The Doorenbos and Kassam/FAO method was applied to estimate the potential and actual yield. It was necessary to use a temperature dependent factor to correct incoherent estimates provide by Doorenbos and Kassam method. Historical series of meteorological data ranging from 10 to 40 years were used to identify the periods of the year with low risks to sorghum crop, besides the probabilities of losses to each sowing date. In Manduri, Piracicaba, Ilha Solteira and Ribeirão Preto, the loss risk is minimized when sowing is made between 15 October and 15 November, with losses lower than 5\%. For all regions, yeld losses below $5 \%$ observed in more than $30 \%$ of the years.

Key words: Sorghum bicolor, water balance, sowing date, climatic risk.

\section{Introdução}

A cultura do sorgo vem ganhando importância no Estado de São Paulo principalmente para semeadura na safrinha desde o início dos anos 90, como alternativa ao milho, devido à sua boa tolerância ao estresse hídrico e baixo custo de produção (SAWASAKI, 1998).

A definição das melhores épocas de semeadura pode ser obtida utilizando-se técnicas convencionais de experimentação, nas quais faz-se a semeadura da cultura em diferentes datas, avaliando-se suas características biométricas e a produtividade alcançada. O custo relativamente elevado e o longo prazo para a consecução deste tipo de pesquisas são alguns dos aspectos que podem ser contornados através da modelagem agrometeorológica, permitindo simular o desenvolvimento da cultura ao longo do tempo, oferecendo resultados consistentes, com baixo custo e prazo relativamente curto.
O objetivo deste trabalho foi parametrizar o modelo de Doorembos e KASSAM (1979) para estimativa da produtividade do sorgo granífero e o risco associado a cada época de semeadura em quatro localidades do Estado de São Paulo: Manduri, Piracicaba, Ribeirão Preto e Ilha Solteira.

\section{Material e Métodos}

O trabalho baseou-se em séries históricas de dados de temperatura do ar $\left({ }^{\circ} \mathrm{C}\right)$ média, mínima e máxima; insolação (horas) e precipitação $(\mathrm{mm})$ das localidades de Manduri, Piracicaba, Ribeirão Preto e Ilha Solteira, no Estado de São Paulo, apresentados na tabela 1. A radiação solar global $\left(\mathrm{MJ} \mathrm{m}^{-2} \mathrm{~d}^{-1}\right)$ foi estimada a partir dos dados de insolação com a equação de Angström-Prescott utilizando-se os coeficientes a e b apresentados na tabela 1 e apresentados por Pereira et al. (2002).

Tabela 1. Municípios analisados com as respectivas coordenadas geográficas, período de coleta de dados, número de anos efetivamente utilizados e valores dos coeficientes a e b da equação de Angström-Prescott

\begin{tabular}{lccccccc}
\hline Localidade & Latitude & Longitude & Altitude & Período & Anos & a & b \\
\hline & & graus & & $\mathrm{m}$ & & & \\
Ilha Solteira $^{(1)}$ & $20^{\circ} 22^{\prime} \mathrm{S}$ & $51^{\circ} 22^{\prime} \mathrm{W}$ & 335 & $1993-2003$ & 10 & 0,24 & 0,52 \\
Manduri $^{(2)}$ & $23^{\circ} 10^{\prime} \mathrm{S}$ & $47^{\circ} 48^{\prime} \mathrm{W}$ & 589 & $1962-1995$ & 34 & 0,25 & 0,50 \\
Piracicaba $^{(3)}$ & $22^{\circ} 42^{\prime} \mathrm{S}$ & $47^{\circ} 38^{\prime} \mathrm{W}$ & 546 & $1951-2002$ & 44 & $(4)$ & $(5)$ \\
Ribeirão Preto $^{(2)}$ & $22^{\circ} 11^{\prime} \mathrm{S}$ & $47^{\circ} 48^{\prime} \mathrm{W}$ & 621 & $1965-1998$ & 30 & 0,13 & 0,73 \\
\hline
\end{tabular}

Fontes dos dados: $\left({ }^{1}\right)$ UNESP/Faculdade de Engenharia de Ilha Solteira. $\left({ }^{2}\right)$ IAC/Centro de Ecofisiologia e Biofísica. $\left({ }^{3}\right)$ USP/Escola Superior de Agricultura "Luiz de Queiroz". $\left({ }^{4}\right)$ Utilizou-se a $=0,28$ para o período outono-inverno e a $=0,25$ para primavera-verão. $\left({ }^{5}\right)$ Utilizou-se $\mathrm{b}=0,51$ para outono-inverno e $\mathrm{b}=0,50$ para primavera-verão (Blanco e Sentelhas, 2002). 
A produtividade potencial (Pp) foi estimada segundo o método da Zona Agroecológica (Doorenbos e KASSAM, 1979), pressupondo que as exigências hídricas, nutricionais e fitossanitárias da cultura sejam atendidas, e que a produtividade seja condicionada apenas pelo potencial genético da cultura e pela radiação solar, fotoperíodo e temperatura do ar (DE WIT, 1965). O método das Zonas Agroecológicas foi parametrizado segundo Doorenbos e KASSAM (1979), especialmente no que concerne aos valores do índice de colheita $(\mathrm{IC}=0,30)$ e para umidade residual do produto $(\mathrm{U}=13 \%)$. Os demais parâmetros utilizados para a aplicação do método estão apresentados na tabela 2 .

Os valores de Pp estimados foram corrigidos por um fator dependente da temperatura do ar $(\mathrm{Ft})$ segundo as recomendações de ArKIN et al. (1976), conforme as equações 1,2 e 3 .

$$
\begin{array}{ll}
F t=-0,25+0,05 \text { TMax } & \mathrm{p} / 5{ }^{\circ} \mathrm{C} \leq \operatorname{Tmax} 25^{\circ} \mathrm{C} \\
F t=1 & \mathrm{p} / 25^{\circ} \mathrm{C} \leq \operatorname{Tmax} 40^{\circ} \mathrm{C} \\
F t=9-0,02 \text { TMax } & \mathrm{p} / \operatorname{Tmax} \leq 40{ }^{\circ} \mathrm{C}
\end{array}
$$

em que: Tmax é a temperatura máxima do ar $\left({ }^{\circ} \mathrm{C}\right)$.

A produtividade real (Pr) foi estimada a partir da deficiência hídrica relativa (1-ETr/ETc) e ponderada por um fator de sensibilidade da cultura ao déficit hídrico (ky), conforme apresentado por Doorenbos e Kassam (1979):

$$
\frac{\operatorname{Pr}}{P p}=\left[1-k y\left(1-\frac{E T r}{E T c}\right)\right],
$$

em que: ETr é a evapotranspiração real da cultura; ETc é a evapotranspiração máxima da cultura, dada pelo produto entre a evapotranspiração de referência (ETo), estimada pelo método de CAMARGO (1971) ajustado segundo CAMARGo et al. (1999), e o coeficiente de cultura $(\mathrm{kc})$ para cada fase fenológica; ky é um fator de sensibilidade ao estresse hídrico, variável com a fase fenológica da cultura. Utilizaram-se os valores de ky propostos por Doorenbos e Kassam (1979) apresentados na tabela 2 .

Tabela 2. Valores de coeficiente de sensibilidade a seca (Ky), capacidade de água disponível (CAD) e índice de área foliar (IAF)

\begin{tabular}{lccc}
\hline Fase fenológica & $\mathrm{ky}$ & CAD & IAF \\
\cline { 2 - 4 } & & $\mathrm{mm}$ & \\
Estabelecimento & 0,2 & 20 & 0,2 \\
Des. vegetativo & 0,6 & 60 & 3,0 \\
Florescimento & 0,7 & 80 & 5,0 \\
Frutificação & 0,6 & 80 & 4,5 \\
Maturação & 0,2 & 80 & 4,0 \\
\hline
\end{tabular}

Os valores de kc e somatório de graus-dia para a conclusão do ciclo da cultura (GD) foram obtidos a partir do trabalho de Costa et al. (1994), utilizandose como referência a cultivar BRS300. Admitindo-se uma temperatura-base de $10{ }^{\circ} \mathrm{C}$ e baseando-se em dados de dois ciclos de cultivo - um de inverno e outro de verão - obteve-se soma térmica de $1.574{ }^{\circ} \mathrm{C}$.dia para o período compreendido entre a germinação e o ponto de maturidade fisiológica.

Os elementos do balanço hídrico foram estimados pelo método de THorthwaite e Mather (1955), considerando-se a capacidade de água disponível (CAD) e o valor de kc variáveis com as fases fenológicas da cultura (Tabela 2). Foram simuladas as quebras de rendimento $(Q$, em $\%)$ para cada decêndio nas séries avaliadas, convertidas em freqüência relativa para análise dos resultados.

Todas as variáveis analisadas neste trabalho, desde os componentes do balanço hídrico de cultura até os valores de Pp e Pr foram calculadas utilizandose um algoritmo computacional desenvolvido em Quick Basic ${ }^{\mathrm{TM}}$, elaborado para o processamento dos dados em escala decendial. A freqüência de quebra de produtividade para cada decêndio, e que foi agrupada em dados médios mensais, foi calculada utilizando o programa Microsoft Excel ${ }^{\mathrm{TM}}$.

\section{Resultados e Discussão}

Observou-se expressiva variação na duração do ciclo da cultura, resultado das diferenças no regime de temperatura entre os locais analisados (Figura 1). Em Ilha Solteira foram observados os menores ciclos de cultivo, com duração entre 88 e 120 dias para semeaduras realizadas em $1^{\circ}-10$ e $1^{\circ}-5$ respectivamente. Em Manduri, situada ao sul do Estado de São Paulo, obteve-se ciclos superiores a 180 dias para as semeaduras realizadas entre $1 .^{\circ}-4$ e $1 .^{\circ}-5$ e, mesmo nas semeaduras em épocas mais favoráveis, como aquelas realizadas entre outubro e dezembro, o ciclo do sorgo foi superior a 110 dias. Em Piracicaba e Ribeirão Preto os ciclos estimados oscilaram entre 109 e 173 dias.

Em sua concepção básica, o modelo de DOORENBOS e KASSAM (1994) pressupõe que a produção potencial de biomassa é função da temperatura do ar, da irradiância solar e da duração do ciclo da cultura, além de fatores genéticos. Quando associado ao conceito de graus-dia, esse modelo superestima os valores de $\mathrm{Pp}$ em épocas ou locais mais frios, como conseqüência da extensão do ciclo da cultura. Para contornar essa distorção nas estimativas, utilizou-se um fator de correção com base na temperatura do ar (Ft) (ARKIN et al, 1976) como forma de reduzir os valores $\mathrm{Pp}$ entre março e junho, tornando suas estimativas mais realistas. 


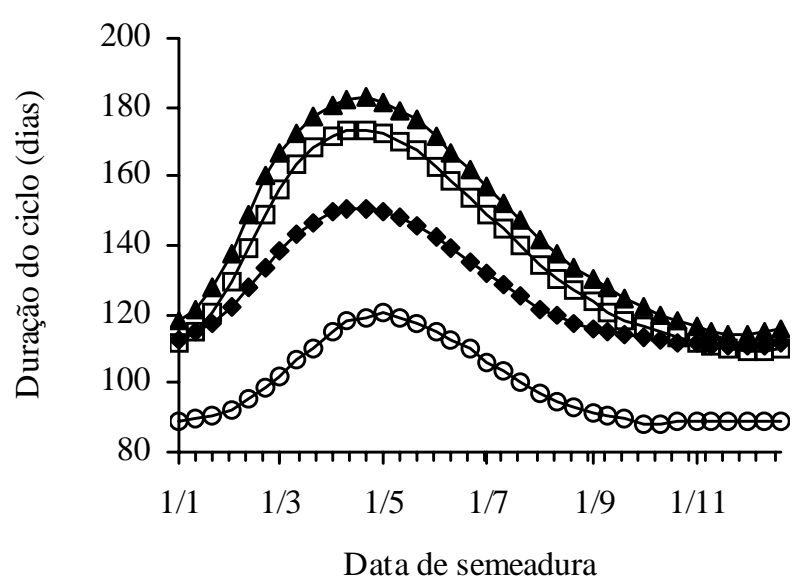

Figura 1. Variação decendial da duração do ciclo da cultura do sorgo, para uma soma térmica de $1574{ }^{\circ} \mathrm{C}$ d, em Manduri ( - ), Piracicaba ( $\square-)$, Ribeirão Preto $(\bullet)$ e Ilha Solteira $(\neg-)$.

As curvas de Pr observadas nas quatro localidades estudadas obedecem em grande parte às curvas anuais da temperatura do ar e do armazenamento hídrico no solo, confirmando a influência desses fatores na determinação da produtividade do sorgo (AgUIAR et al., 2000). Segundo esses autores, a queda na produtividade devida a tais fatores normalmente está relacionada com o decréscimo do número de sementes decorrente da redução do período de desenvolvimento da panícula.

A queda de Pr entre março e maio pode ser atribuída ao frio durante o inverno e à deficiência hídrica intensa, reduzindo expressivamente as estimativas de Pr. Para os quatro locais, nas melhores épocas de semeadura, a produtividade ficou acima de $5.600 \mathrm{~kg} \mathrm{ha}^{-1}$, níveis considerados elevados se comparados com a produtividade normalmente observada em condições de campo. Essa tendência, como destacado por Doorembos e KASSAM (1979), devese ao fato de que as perdas estimadas nesse caso são resultantes apenas da deficiência hídrica e da queda na temperatura, sem considerar perdas decorrentes de desequilíbrios nutricionais, das pragas e doenças e da presença de plantas daninhas. Além disso, o método das Zonas Agroecológicas foi desenvolvido com base em dados de campo, coletados em parcelas experimentais cultivadas com variedades altamente produtivas, o que também contribui para a elevação das estimativas de Pp.

O regime de chuva de cada localidades explica em grande parte a variação das curvas de Q (Figura 3). Em Manduri, têm-se os valores máximos de $Q$ para semeaduras realizadas entre $1 .^{\circ}-4$ e $1 .^{\circ}-6$, porém sempre inferiores a 11\% (Figura 3). Em Ribeirão Preto, onde há um período de seca bem definido entre maio e setembro, obtiveram-se valores máximos de $\mathrm{Q}$ próximos de $15 \%$ entre abril e junho. Para semeadura na safrinha, entretanto, as condições podem ser consideradas favoráveis, com valores de $\mathrm{Q}$ abaixo de 10\%, em média. Em Ilha Solteira, observaram-se os maiores valores de $Q$, devido à redução no volume de chuvas associado às elevadas taxas de evapotranspiração entre abril e agosto. Pela variação decendial da quebra relativa de produtividade (Q) (Figura 3), observa-se que, para todas as localidades, o período compreendido entre $1 .^{\circ}-10$ e $1 .^{\circ}-12$ é o que o oferece menores riscos de perdas e o primeiro decêndio de novembro, o menor risco de perda em Manduri, Piracicaba e Ribeirão Preto, com perdas médias menores que $4 \%$. Em Ilha Solteira, a época de semeadura com menor risco de perda de rendimento é $1 .{ }^{\circ}-12$, com $Q=2,6 \%$.

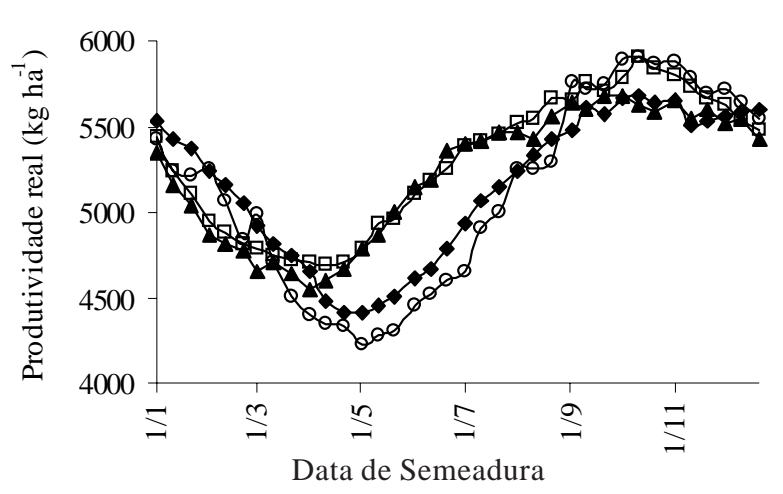

Figura 2. Variação decendial da produtividade real estimada para Manduri ( $\_-$), Piracicaba $(\square-)$ ), Ribeirão Preto $(\multimap-)$ e Ilha Solteira $(-\circ)$.

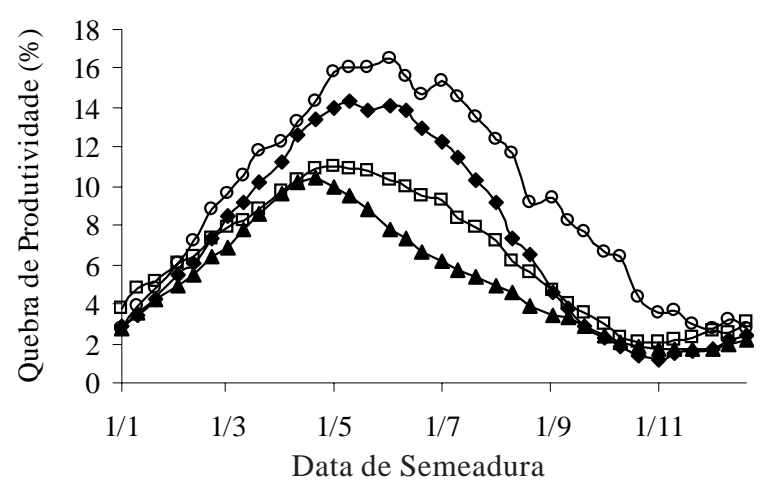

Figura 3. Valores médios da quebra relativa de produtividade para a cultura do sorgo, para semeaduras feitas nos 36 decêndios do ano em Manduri (־) ), Piracicaba $(-\square)$, Ribeirão Preto $(\multimap)$ e Ilha Solteira $(-\circ)$. 
As freqüências médias mensais para diferentes faixas de $Q$ nos locais analisados são apresentados na Tabela 3. Em Manduri, perdas acima de $5 \%$ em semeaduras realizadas entre outubro e dezembro ocorreram em menos de $10 \%$ dos casos simulados. Para as semeaduras realizadas em fevereiro, em $42 \%$ dos anos simulados, os valores de Q ficaram abaixo de 5\%. Em Piracicaba, a distribuição de freqüência de $Q$ para a safra das águas é semelhante ao observado em Manduri, havendo aumento nos riscos para a cultura durante a safrinha.
Os riscos de perda de rendimento em fevereiro e março foram relativamente elevados, com perdas superiores a $20 \%$ em cerca de $20 \%$ das simulações. Ribeirão Preto tem condições semelhantes às verificadas em Piracicaba, porém com um período favorável à cultura entre outubro e janeiro e perdas maiores para semeaduras realizadas fora deste período. Em Ilha Solteira, verificaram-se condições favoráveis à cultura entre novembro e janeiro, mas com boas condições para a semeadura em fevereiro. (Tabela 3).

Tabela 3. Freqüência de perda de produtividade (em \%) para diferentes faixas de quebra de produtividade, no Estado de São Paulo

\begin{tabular}{|c|c|c|c|c|c|c|c|c|c|c|c|}
\hline \multicolumn{7}{|c|}{ Manduri } & \multicolumn{5}{|c|}{ Ribeirão Preto } \\
\hline \multirow{2}{*}{ Mês } & \multicolumn{5}{|c|}{ Perda de Produtividade } & \multirow[b]{2}{*}{ Mês } & \multicolumn{5}{|c|}{ Perda de Produtividade } \\
\hline & 0 a 5 & 6 a 10 & 11 a 15 & 15 a 20 & $>20$ & & 0 a 5 & 6 a 10 & 11 a 15 & 15 a 20 & $>20$ \\
\hline & \multicolumn{5}{|c|}{$\%$} & \multicolumn{6}{|c|}{$\bar{L} \%$} \\
\hline Janeiro & 69,6 & 25,5 & 4,9 & 0,0 & 3,9 & Janeiro & 83,3 & 15,6 & 1,1 & 0,0 & 0,0 \\
\hline Fevereiro & 42,2 & 41,2 & 13,7 & 2,9 & 2,9 & Fevereiro & 38,9 & 37,8 & 20,0 & 3,3 & 0,0 \\
\hline Março & 19,6 & 51,0 & 23,5 & 5,9 & 9,8 & Março & 11,1 & 34,4 & 33,3 & 12,2 & 8,9 \\
\hline Abril & 16,7 & 35,3 & 24,5 & 15,7 & 19,6 & Abril & 0,0 & 16,7 & 25,6 & 38,9 & 18,9 \\
\hline Maio & 21,6 & 29,4 & 30,4 & 13,7 & 31,4 & Maio & 1,1 & 7,8 & 32,2 & 25,6 & 33,3 \\
\hline Junho & 32,4 & 37,3 & 25,5 & 2,0 & 24,5 & Junho & 3,3 & 24,4 & 24,4 & 22,2 & 25,6 \\
\hline Julho & 51,0 & 35,3 & 8,8 & 2,0 & 16,7 & Julho & 16,7 & 31,1 & 20,0 & 23,3 & 8,9 \\
\hline Agosto & 71,6 & 20,6 & 4,9 & 0,0 & 7,8 & Agosto & 40,0 & 34,4 & 18,9 & 4,4 & 2,2 \\
\hline Setembro & 84,3 & 12,7 & 0,0 & 0,0 & 2,9 & Setembro & 75,6 & 21,1 & 2,2 & 1,1 & 0,0 \\
\hline Outubro & 94,1 & 2,9 & 1,0 & 1,0 & 2,9 & Outubro & 97,8 & 2,2 & 0,0 & 0,0 & 0,0 \\
\hline Novembro & 91,2 & 8,8 & 0,0 & 0,0 & 1,0 & Novembro & 96,7 & 3,3 & 0,0 & 0,0 & 0,0 \\
\hline Dezembro & 69,6 & 25,5 & 4,9 & 0,0 & 2,9 & Dezembro & 83,3 & 15,6 & 1,1 & 0,0 & 0,0 \\
\hline \multicolumn{7}{|c|}{ Piracicaba } & \multicolumn{5}{|c|}{ Ilha Solteira } \\
\hline \multirow{2}{*}{ Mês } & \multicolumn{5}{|c|}{ Perda de Produtividade } & & \multicolumn{5}{|c|}{ Perda de Produtividade } \\
\hline & 0 a 5 & 6 a 10 & 11 a 15 & 16 a 20 & $>20$ & Mês & 0 a 5 & 6 a 10 & 11 a 15 & 15 a 20 & $>20$ \\
\hline & \multicolumn{5}{|c|}{$\bar{\nu} \%$} & & \multicolumn{5}{|c|}{$\overline{0} \%$} \\
\hline Janeiro & 76,0 & 18,6 & 4,7 & 0,8 & 15,8 & Janeiro & 36,7 & 43,3 & 20,0 & 0,0 & 0,0 \\
\hline Fevereiro & 57,4 & 18,6 & 16,3 & 7,8 & 19,2 & Fevereiro & 30,0 & 43,3 & 23,3 & 3,3 & 0,0 \\
\hline Março & 34,9 & 34,9 & 20,2 & 6,2 & 26,7 & Março & 24,1 & 27,4 & 13,7 & 31,1 & 3,7 \\
\hline Abril & 17,1 & 31,0 & 38,0 & 9,3 & 50,8 & Abril & 7,4 & 22,2 & 14,8 & 40,7 & 14,8 \\
\hline Maio & 20,2 & 34,9 & 26,4 & 11,6 & 43,3 & Maio & 0,0 & 7,4 & 7,4 & 29,6 & 55,6 \\
\hline Junho & 29,5 & 41,1 & 20,2 & 5,4 & 34,2 & Junho & 0,0 & 0,0 & 3,7 & 11,1 & 85,2 \\
\hline Julho & 42,1 & 42,1 & 9,5 & 5,6 & 31,7 & Julho & 0,0 & 7,4 & 7,4 & 3,7 & 81,5 \\
\hline Agosto & 65,9 & 26,2 & 6,3 & 1,6 & 17,5 & Agosto & 11,1 & 0,0 & 7,4 & 22,2 & 59,3 \\
\hline Setembro & 85,7 & 12,7 & 1,6 & 0,0 & 7,5 & Setembro & 0,0 & 7,4 & 3,7 & 25,9 & 63,0 \\
\hline Outubro & 95,2 & 4,8 & 0,0 & 0,0 & 2,5 & Outubro & 0,0 & 0,0 & 22,2 & 14,8 & 63,0 \\
\hline Novembro & 94,4 & 5,6 & 0,0 & 0,0 & 2,5 & Novembro & 7,4 & 11,1 & 14,8 & 25,9 & 40,7 \\
\hline Dezembro & 76,0 & 18,6 & 4,7 & 0,8 & 4,2 & Dezembro & 36,7 & 43,3 & 20,0 & 0,0 & 0,0 \\
\hline
\end{tabular}




\section{Referências}

AGUIAR, L.M.S.; MORAIS, A. V.C.; GUIMARÃES, D.P. Cultivo do sorgo - Clima. Embrapa Milho e Sorgo, Sistema de Produção, n.2, 2000. Disponível em: http:// www.cnpms.embrapa.br/publicacoes/sorgo/clima.htm. Acesso em 10/11/2005.

ARKIN, G.F.; VANDERLIP, R.L.; RITCHIE, J.T. A. dynamic grain sorghum growth model. Transactions of the ASAE, St. Joseph, v.19, p.622-630. 1976.

BLANCO, F.F.; SENTELHAS, P.C. Coeficientes da equação de angströn-Prescott para estimativa da insolaçãoo para Piracicaba, SP. Revista Brasileira de Agrometeorologia, Santa Maria, v. 10, n. 2, p. 295-300, 2002.

CAMARGO, A.P. Balanço hídrico no Estado de São Paulo. Campinas: IAC, 1971. 24 p. (Boletim Técnico, 116)

CAMARGO, A.P.; MARIN, F.R.; SENTELHAS, P.C.; PICINI, A.G. Ajuste da equação de Thornthwaite para estimar a evapotranspiração potencial em climas áridos e superúmidos, com base na amplitude térmica diária. Revista Brasileira de Agrometeorologia, Santa Maria, v.7, n.2, p.251-257, 1999.
COSTA, E.F.; COUTO, L.; SANS, L.M.A.; NETO, M.S.A.; BRITO, R.A.L. Estimativa do consumo de água pela cultura do sorgo (Sorghum bicolor L.), a partir da evapotranspiração de referência na região de Sete Lagoas, MG. Irrigação e Drenagem, v.3, p.1-11, 1994.

DOORENBOS, J.; KASSAM, A.H. Yiels response to water. Rome: FAO, 1979. 306p. (FAO: Irrigation and Drainage Paper, 33).

PEREIRA, A.R.; ANGELOCCI, L.R.; SENTELHAS, P.C. Agrometeorologia: fundamentos e aplicações práticas. Guaíba: Agropecuária, 2002. 478p.

SAWAZAKI, E. Sorgo granífero. In: FAHL, J.L. et al. (Ed.). Instruções agrícolas para as principais culturas econômicas. Campinas: Instituto Agronômico, 1998. p.46-47. (Boletim Técnico IAC, 200)

THORNTHWAITE, C.W.; MATHER, R.J. The water balance. New Jersey: Laboratory of Climatology, 1955. 104 p. (Publications of Climatology, v.8, n.1) 